\title{
Social and Behavioral Characteristics of Young Adult Drink/Drivers Adjusted for Level of Alcohol Use
}

\author{
C. Raymond Bingham, Michael R. Elliott, and Jean T. Shope
}

\begin{abstract}
Background: Alcohol consumption and drink/driving are positively correlated and many predictors of alcohol use also predict drink/driving. Past research has not fully distinguished the contributions of personal risk factors from the level of alcohol use in the prediction of drink/driving. As a result, the extent to which predictors are specific to drink/driving, versus due to a mutual association to alcohol use, is unclear.

Methods: This study examined the unique and shared risk factors for drink/driving and alcohol use, and examined the attributable risk (AR) associated with predictors of drink/driving while adjusting for alcohol use. Study data were from a telephone survey of 3,480 Michigan-licensed young adults who were drinkers. Four groups of drink/drivers were formed based on the prior 12-month maximum severity of drink/driving: (1) never drink/driving; (2) driving at least once within an hour of 1 or 2 drinks; (3) driving within an hour of 3 or more drinks or while feeling the effects of alcohol; and (4) drinking while driving.

Results: Lower perceived risk of drink/driving, greater social support for drinking and drink/ driving, greater aggression and delinquency, more cigarette smoking, and more risky driving behaviors uniquely predicted drink/driving severity in models adjusted for alcohol use. The largest ARs were associated with social support for drinking and drink/driving and perceived risk of drink/driving.

Conclusions: These results confirm that alcohol use and drink/driving share risk factors, but also indicate that part of the variation in these factors is specific to drink/driving. Implications for interventions to reduce drink/driving are discussed.
\end{abstract}

Key Words: Drink-Driving, Population-Attributable Risk, Young Adults, Risk-Factors, Driving Behaviour, Alcohol Use.

$\mathrm{D}$ RIVING UNDER THE influence of alcohol contributes significantly to the occurrence of motor vehicle crashes and resulting injuries and fatalities, and is a serious public health concern. Alcohol-related motor vehicle crash is defined as involving at least 1 driver or nonoccupant (e.g., pedestrian, pedalcyclist) who has a minimum blood alcohol concentration (BAC) of $0.01 \mathrm{~g} / \mathrm{dL}$. In 2004, there were 16,694 alcohol-related crash fatalities, representing $39 \%$ of all traffic fatalities that year. Young adult drivers aged from 21 to 24 have the highest rate of fatal alcohol-related motor vehicle crashes, accounting for $31 \%$ of all such incidents (National Highway Traffic Safety Administration, 2005). For many years, reducing the rate of driving under the influence of alcohol (drink/driving) has been a high priority in transportation safety, and as a result, rates of drink/driving have

From the University of Michigan Transportation Research Institute, Ann Arbor, Michigan (CRB, JTS); and the Department of Biostatistics, University of Michigan, Ann Arbor, Michigan (MRE).

Received for publication July 28, 2006; accepted December 23, 2006.

Reprint requests: C. Raymond Bingham, University of Michigan Transportation Research Institute,Ann Arbor, MI; E-mail: rbingham@, umich.edu

Copyright (C) 2007 by the Research Society on Alcoholism.

DOI: 10.1111/j.1530-0277.2007.00350.x consistently declined. However, this decline has recently plateaued (Williams, 2006), pointing to the need for a greater understanding of the characteristics of drink/drivers so that the effectiveness of programs and interventions to reduce drink/driving can be increased.

\section{ALCOHOL USE AND DRINK/DRIVING}

Alcohol use and drink/driving are positively associated. Consistent evidence indicates that the level of alcohol consumption is associated with drink/driving and with the severity of drink/driving outcomes (Escobedo et al., 1995; Grasmick et al., 1993; Grube and Voas, 1996; Howland and Hingson, 1987; Lee et al., 1997; McMillen et al., 1992; Padilla and Morrisey, 1993; Sabel et al., 2004; Treno et al., 1996). Drink/drivers who are injured in a crash are more likely than injured nondrink/drivers to perceive that they have a drinking problem, to have a higher frequency of intoxication in the month before the crash, and to report a greater frequency of drink/driving (Vingilis et al., 1994).

Drinking location and, although found inconsistently in the literature (Gruenewald et al., 1996), beverage type have been shown to predict drink/driving (Greenfield and Rogers, 1999; Lee et al., 1997). However, the context of drinking, specifically drinking location, is a stronger pre- 
dictor in some research than is beverage type or amount of alcohol consumed, with locations that require transportation (e.g., restaurants and bars) predicting drink/driving (Gruenewald et al., 1996). These ecological characteristics of drinking may seem separate from the individual characteristics that are associated with drink/driving, but may not be. The same individual characteristics that motivate drinking patterns may also affect the choice of drinking venue. Such an association is not investigated in the current study, but should be examined more extensively by future studies.

\section{PERSONALITY-RELATED FACTORS}

Personality traits and related factors are associated with risky driving behaviors (Burns and Wilde, 1995; Iversen and Rundmo, 2002; Jonah, 1997; Patil et al., 2006; Ulleberg, 2001), including drink/driving (McMillen et al., 1991, 1992). Drink/drivers have been found to possess greater hostility and psychopathic deviance (McMillen et al., 1991), sensation seeking (Donovan et al., 1985), and risk taking (McMillen et al., 1992; Patil et al., 2006) than nondrink/drivers. While this past research provides an insight into personality characteristics related to drink/ driving, it does little to disentangle the influence of these characteristics from those of alcohol use on drink/driving. This study adds clarity to this issue.

\section{PERCEPTION AND ATTITUDES}

The influence of personality on driving behavior appears to be mediated through attitudes and perceptions (Turrisi et al., 1997; Ulleberg and Rundmo, 2003), and attitudes toward traffic safety have been found to correlate with risky driving behavior, including drink/driving (Baum, 2000; Greenberg et al., 2005). Perceptions of the degree of risk associated with drink/driving, perceptions of peers' relative approval/disapproval of drink/driving, extent of self-control over drinking, control over the external environment, and willingness to commit driving violations also predict drink/driving (Donovan, 1993; Donovan et al., 1985; Greenfield and Rogers, 1999; Grube and Voas, 1996). However, as with personality-related factors, research has not clarified the role of alcohol use in the association between expectations and attitudes, and drink/ driving behavior.

\section{PROBLEM BEHAVIORS}

Aggressive and deviant behavior, illegal substance use, and cigarette smoking have all been related to at-risk alcohol use and drink/driving. Smoking cigarettes and using drugs has also been shown to be higher among drink/drivers (Everett et al., 1999). Other factors involved in this association are likely to include greater risk of injury due to drink/driving, heavy smoking, heavy alcohol consumption (Donovan et al., 1985; Shepherd et al., 2004), and driving anger (Ulleberg, 2001). Greater involvement with family, community, and school appears to be a protective factor for teens through their association with lower levels of drink/driving and riding with drink/drivers (Sabel et al., 2004).

\section{DRIVING BEHAVIOR}

Research indicates that drink/drivers are less likely than other drivers to adopt safe driving behaviors, and are more likely to be risky drivers, even when sober, than are nondrink/drivers. Higher levels of drink/driving are associated with lower rates of safety belt use. In addition, drink/driving is associated with other high-risk driving behaviors, including speeding, riding with a drink/driver, drug/driving, and aggressive driving (Bingham and Shope, 2004; Donovan, 1993; Everett et al., 1999; McMillen et al., 1992; Patil et al., 2006; Sabel et al., 2004; Shope and Bingham, 2002; Yu et al., 2004).

\section{PURPOSE}

The purpose of this study was to distinguish alcohol consumption from personality, attitudinal, and behavioral risk factors in the prediction of drink/driving. Sex differences were examined by estimating the models separately for men and women. As just reviewed, more alcohol use, whether measured in amount consumed or patterns (i.e., where, when, with whom), is related to more drink/driving (Gruenewald et al., 1996). Furthermore, many individual risk factors are associated with both alcohol use and drink/ driving; however, it is unclear to what extent this association results from the mutual prediction of drink/driving by individual risk factors and alcohol use versus confounding due to the close association between these 2 factors. There are 3 patterns of association that might occur between individual characteristics, alcohol use, and drink/driving (see Fig. 1). The first is an unconfounded association in which individual characteristics and alcohol use both independently predict drink/driving (see Fig. 1A). In this case, the associations between these 2 variables and drink/ driving would be the same when considered in separate models and together in the same model. The other 2 types of association could result in confounded results or misinterpretations of the associations among these variables. Partial confounding (Fig. 1B) occurs when individual risk factors and alcohol use both predict drink/driving, but part of the association between alcohol use and drink/ driving is due to the association between individual characteristics and drink/driving. In this case, the association between individual characteristics and drink/driving is attenuated when alcohol use is in the model. Finally, complete confounding occurs when individual risk factors and alcohol use both appear to predict drink/driving, but the association between alcohol use and drink/driving is completely due to the association between individual risk factors and alcohol use (see Fig. 1C). In this case, the 


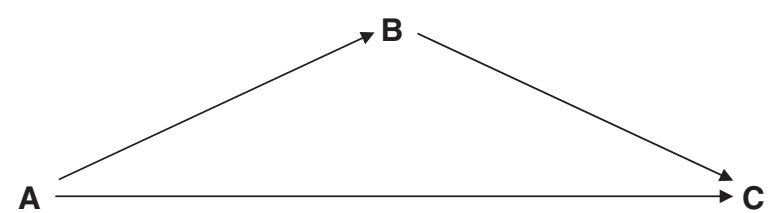

(A) Mutual Prediction

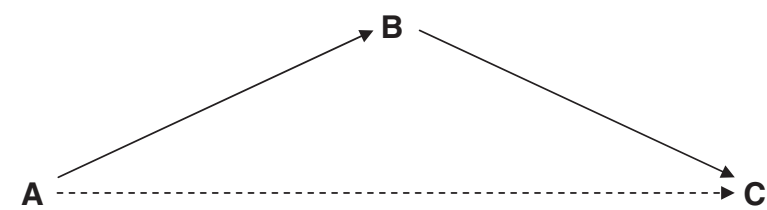

(B) Partial Confounding

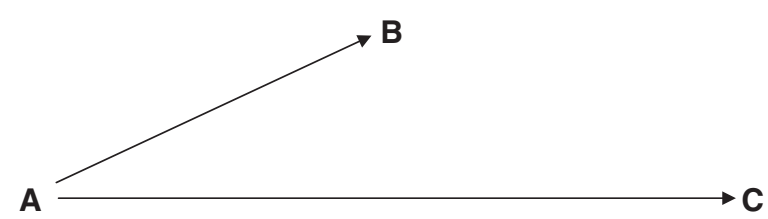

(C) Complete Confounding

Fig. 1. Potential patterns of association among individual characteristics, alcohol use, and drink/driving.

association between alcohol use and drink/driving would not be significant if individual risk factors are included in the model. This study examined the risk factors reported in past research to be associated with both alcohol use level and drink/driving and attempted to determine the extent to which these associations resulted from mutual prediction versus some level of confounding due to the association between alcohol use and drink/driving.

\section{METHODS}

\section{Sample}

The study data were collected from participants in a telephone survey administered in 1999 and 2000 as part of an on-going longitudinal study. The longitudinal study began when participants were in the fifth and sixth grades as part of 2 intervention evaluation studies called the Alcohol Misuse Prevention Study (AMPS) and the Genessee Intermediate School District (GISD) study. Enrollment began in fifth and sixth grades with students from school districts in southeastern Michigan, and the intervention was conducted when participants were in the fifth/sixth, seventh/eighth, and 10th grades with follow-up school surveys in the 10th and 12th grades in high school. The AMPS and GISD data measured demographics, substance use, perceived parental attitudes and behavior, and other psychosocial variables. The effectiveness of the AMPS and GISD is reported elsewhere (Shope et al., 1992, 1996, 2001). Recent analyses examining intervention group differences in drink/driving during young adulthood showed no effect.

In 1999, when they averaged 24.3 years of age ( $\mathrm{SD}=0.8)$, young adult AMPS and GISD participants who had current Michigan driver licenses were followed up with a telephone interview. Licensure status and addresses of eligible participants were obtained from the Michigan Department of State, and processed for tracking and interviewing. Advance letters invited participants to be part of the young adult follow-up, and offered $\$ 10$ for completing the interview, and an additional $\$ 5$ if they sent their telephone number to project staff by phone, e-mail, or conventional mail. The interview and study were described to the participants, and their participation constituted consent. The participants were harder to track and contact than anticipated. Once contacted, however, only $6 \%$ refused the interview and a total of 4,230 completed the interview $(58.5 \%$ of the original sample based on definition RR5, American Association for Public Opinion Research, 2000). Interviews were conducted by trained personnel using computer-based interviews that provided immediate data entry.

Respondents and nonrespondents were compared using measures from 10th- and 12th-grade high school surveys and data from State of Michigan driver history records to check for nonparticipation bias. There were some significant differences associated with very small effects ranging from $d=-0.009$ for alcohol availability in the 12 th grade to $d=0.370$ for 10 th-grade marks in school. Only differences in age ( $d=0.267$; nonrespondents older), 10th-grade marks in school ( $d=0.370$, nonrespondents had lower marks), and living with both biological parents at 12 th grade $(d=-0.203$; respondents were more likely to live with both biological parents) were large enough to be considered small effects (see Cohen, 1992). The core items of the study's analysis, namely alcohol misuse, cigarette use, marijuana use, and driving behavior, did not differ between respondents and nonrespondents.

The study sample includes 3,480 respondents in a single young adult telephone survey who reported that they drank alcohol in the year before the interview. Participants in this study were $48.3 \%$ male ( $n=1,681$ men, $n=1,799$ women) and $88.0 \%$ white, $74.2 \%$ never married, $83.6 \%$ employed, $27.1 \%$ students, and had been licensed to drive for an average of 8.1 years $(S D=1.1$ years, range $=2-12)$ when they completed the telephone survey. These subjects are reasonably representative of their age cohort in the general Michigan population in race, marital status, employment, and student status. A recent by-gender comparison of Michigan state driving records for drivers from the study sample and nonsample same-age drivers demonstrated a high degree of similarity in the proportions of drivers with no offense, 1 offense, and 2 or more offenses and crashes (Elliott et al., 2000).

\section{MEASURES}

\section{Background and Alcohol Use Measures}

Demographic Characteristics. Personal annual income, age (years at interview), marital status (ever vs never), and completed education were included in the models as control variables. All of these variables have been shown to predict drink/driving, and were selected as controls in this study for this reason. Personal income was coded as $1=$ under $\$ 5,000$, $2=\$ 5,000$ to $\$ 14,999,3=\$ 15,000$ to $\$ 24,999,4=\$ 25,000$ to $\$ 34,999,5=\$ 35,000$ to $\$ 44,999,6=\$ 45,000$ to $\$ 54,999$, and $7=\geq \$ 55,000$. Completed education was the highest level of education completed at the time of the survey: $1=<$ eighth grade, $2=$ finished eighth grade, $3=$ some high school, $4=$ graduated high school, $5=$ graduated technical or trade school, $6=$ some college, $7=$ graduated college, $8=$ some graduate or professional school, and $9=$ earned a graduate or professional degree. Race/ethnicity was not included, as the sample, like the schools where the sample was originally recruited, is predominantly white.

Alcohol Use Measures. No single measure is adequate to represent the complex behavioral variation in individual alcohol use. For this reason, we chose 3 measures to use in conjunction with each other. While 3 measures are better 
than 1 , it cannot be assumed that these 3 measures completely measure the construct of alcohol use.

Quantity-frequency (QF) of alcohol consumption was the product of 2 items: frequency of alcohol consumption (ranged from $0=$ never to $4=4$ or more times a week) and number of drinks consumed on a typical drinking day in the previous year (ranged from $0=$ never drink alcohol, to $5=10$ or more). These 2 items were developed for this study, but were modeled after quantity and frequency of drinking items commonly used in alcohol research, and that are available at http://lib.adai.washington.edu/instruments/.

The 10-item Alcohol Use Disorders Identification Test (AUDIT; Babor et al., 1992) includes 3 items measuring consumption patterns, 3 items measuring alcohol dependence, and 4 measuring the personal/social problems caused by alcohol. The AUDIT was developed as a screening tool, but has been used widely in research and epidemiological studies. Alcohol Use Disorders Identification Test scores were calculated by summing across the 10 items. Internal consistency was good $(\alpha=0.77)$.

Alcohol consequences (Donovan, 1993) measured how frequently in the prior 12 months drinking had resulted in: a hangover; physical illness or passing out; difficulties with a close friend, spouse, or partner; problems on the job; trouble with the police; injury to themselves or others; inability to stop drinking once started; failure to do what was normally expected; inability to remember the night before; an alcohol-involved accident; or receiving expressions of concern from family, friends, or physician about drinking. A summary score was calculated by standardizing the responses $(M=0, \mathrm{SD}=1)$, calculating the mean across items and adding the absolute value of the lowest score so that the low value was zero $(\alpha=0.76$, has demonstrated construct and predictive validity).

\section{Intraindividual Factors}

Personality/Hostility. Risk-taking propensity was measured by 4 items (Donovan, 1993). An example item is "I enjoy the thrill I get when I take risks." Responses were $1=$ not at all like me to $3=$ a lot like me. Overall scores were calculated as the mean response to the 4 items, with a higher score indicating a greater risk-taking propensity $(\alpha=0.72$, has demonstrated construct and predictive validity).

Hostility was measured by 7 items that were developed for this study. Participants were asked to rate how well each of the items described them. An example item is "When I lose my temper I've been known to hit or slap someone." Participants indicated how well each item described them on a scale from $1=$ not at all like me to $3=\mathrm{a}$ lot like me. Higher mean scores indicated greater hostility. Factor analyses show that this measure is unidimensional. The internal consistency for this measure is relatively low, $\alpha=0.54$ (see Cattell, 1982); however, it has performed well as a predictor of driving behavior in past research.

Tolerance of deviance (TOD) was measured by 10 items (Donovan, 1993) rating the wrongness of specific behaviors. An example item is "To start a fight or hit someone." The responses ranged from $1=$ very wrong to $4=$ not at all wrong. An overall mean score was calculated so that higher values indicated greater TOD $(\alpha=0.79$, has demonstrated construct and predictive validity).

Competitive attitude toward driving (Donovan, 1993) was measured by asking how strongly participants agreed/disagreed with 5 statements. An example item from this scale is "It's fun to beat other drivers when the lights change." Responses ranged from $1=$ strongly agree, $2=$ agree, $3=$ disagree, and $4=$ strongly disagree $(\alpha=0.80$, has demonstrated construct and predictive validity).

Individual/Social Characteristics. The perceived risk of drink/driving was measured as the likelihood that driving within an hour of having 3 (men) or 2 (women) drinks of alcohol would result in: getting stopped for drinking and driving; losing a driver's license; being unable to drive as well as usual; being in a car crash; injuring oneself or others; and receiving a fine. The responses ranged from $1=$ very likely to $4=$ very unlikely. This measure was developed for this study. Factor analyses were conducted, showing that the scale is unidimensional. Scale scores were calculated so that a higher mean score corresponded to greater risk perception $(\alpha=0.91)$.

Social support for drinking and drink/driving consisted of 6 items measuring attitudes toward drink/driving, and the drinking behavior of the participant's friends. The items were standardized $(M=0, \mathrm{SD}=1)$, averaged, and added to the absolute value of the lowest mean score to provide a summary scale with a low value of $0(\alpha=0.80)$. This measure was developed for this research and has demonstrated construct and predictive validity in previous analyses.

Problem Behavior. Delinquent behavior (Donovan, 1993) was measured by a 9-item scale that asked how frequently in the prior 12 months the participant had given a fake excuse for missing work, school, or meetings; damaged public or private property on purpose; started a fight; given false information for a job or loan application; shoplifted something of value; started an argument; damaged something of value to someone else because they were angry at that person; lied to someone they were close to; and taken things of value that did not belong to them. Responses $(1=$ never to $6=10$ times or more often) were averaged to obtain scale scores. Internal consistency was adequate $(\alpha=0.64$; see Cattell, 1982; has demonstrated construct and predictive validity).

Lifetime and past year drug use were measured by asking participants to report whether they had used $(0=$ no, $1=$ yes) each of 10 drugs ever in their lifetime, and in the past year, including marijuana; amphetamines; Quaaludes; tranquilizers; psychedelic drugs; crack or cocaine; opiates; and prescription drugs for nonmedical purposes. 
Scale scores were calculated by separately summing responses to the 9 items measuring lifetime $(\alpha=0.76)$ and past year $(\alpha=0.70)$ drug use. These items were developed for this study.

Cigarette smoking was measured by 3 items asking participants how much they smoked cigarettes. The responses to the 3 items, coded in numbers (e.g., 2), units (e.g., packs), and time intervals (e.g., daily), were used to calculate daily rates of cigarette use (e.g., 40). This measure was developed for this study.

Driving Behavior. Driving under the influence of drugs (drug/driving) was measured by items that asked how many times in the prior 12 months the respondent had driven after: (1) smoking marijuana; or (2) using any of the list of other drugs as above. Responses were recoded ( $1=$ once, $2=$ twice, $3=3$ times, $4=4$ times, $5=5$ times, $6=6-9$ times, $7=10-14$ times, $8=15-19$ times, $9=20-24$ times, $10=25-29$ times, $11=30-49$ times, $12=50-99$ times, and $13=100$ times or more often) (Donovan, 1993), and averaged to obtain scale scores. Internal consistency was adequate $(\alpha=0.60$; see Cattell, 1982).

High-risk driving (Donovan, 1993) was assessed by 20 items that measured exceeding the speed limit, and inappropriate passing, following, lane usage, yielding/ right-of-way, turning, and observance of control signals. Many of the behaviors measured constitute ticketable offenses $(\alpha=0.86$, has demonstrated construct and predictive validity, see Shope and Bingham, 2002).

Risk-taking driving (Donovan, 1993) was measured by 8 items asking how often participants took chances for the fun of it, saw how fast one could drive, out-maneuvered other drivers for the thrill of it, passed cars because it was exciting, drove dangerously for enjoyment, tested their driving skills in ways that others might find risky, took driving risks because it felt good, and tried to beat other drivers at stoplights to impress someone. The responses $(1=$ very often, $2=$ often, $3=$ once in a while, and $4=$ never) were reverse scored and averaged so that a higher score indicated more frequent risk-taking driving $(\alpha=0.86$, has demonstrated construct and predictive validity).

Driving aggression (Donovan, 1993) was measured by 4 items that asked how often participants took chances for the fun of it; honked the horn at drivers who cut in front of them; made rude gestures at drivers who did things that were annoying; and tailgated other drivers to get back at them. Item responses $(1=$ very often, $2=$ often, $3=$ once in a while, and $4=$ never) were reverse scored and averaged so that a higher score indicated more frequent driving aggression. Internal consistency was adequate $(\alpha=0.63$; see Cattell, 1982; has demonstrated construct and predictive validity).

Frequency of safety belt use was measured on short and long drives ( 2 items). Responses $(1=$ always or almost always, $2=$ most of the time, $3=$ sometimes, $4=$ seldom, and $5=$ never or almost never) were reverse scored and averaged so that a higher score indicated more frequent safety belt use $(\alpha=0.91)$. This measure was developed for this study. Factor analyses demonstrate unidimensionality.

Drink/Driving Groups. Six items measured the frequency of drink/driving (Donovan, 1993), and responses were used to form drink/driving groups. The items were, "In the past 12 months how many times did you": "drive within an hour of drinking alcohol"; "drive within an hour of drinking 1 or 2 beers or other alcoholic beverages"; "drive within an hour of drinking 3 or more drinks"; "drive when you felt high or light headed from drinking"; "drive when you knew your drinking may already have affected your coordination"; and "drink in the car while you were driving." Responses, which were given as the actual frequency (range 0-999), were dichotomized into never versus at least once. Participants were classified as those who never drove within an hour of drinking (nondrink/drivers; $n=1,256 ; 779$ women, $43 \%$ of women; 477 men, $28 \%$ of men); drove at least once within an hour of having 1 or 2 drinks (low-drink/drivers; $n=1,100$; 630 women, $35 \%$; 470 men, $28 \%$ ); drove at least once within an hour of 3 or more drinks, when high or light headed, or when their coordination had been affected by drinking (high-drink/drivers; $n=773 ; 281$ women, $16 \%$; 492 men, 29\%), and those who reported they had drank while driving (drank while driving; $n=351 ; 109$ women, $6 \% ; 242$ men, $14 \%$ ).

Self-reported measures of socially proscribed or frankly illegal behaviors are often suspected of lacking validity. However, research supports the validity of self-report of illegal behaviors, including substance use, when confidentiality is protected as it was in this study (Babor and Del Boca, 1992; Babor et al., 2000; Chaikelson et al., 1994; Darke et al., 1991; O’Malley et al., 1983; Williams et al., 1995).

\section{Plan of Analysis}

Multinomial logistic regression models were tested separately for men and women. First, regression models were estimated separately for each domain of risk factors, and then these same models were adjusted for alcohol use. Second, all risk factors were entered into the model simultaneously to obtain overall unadjusted estimates, and then the model was tested a second time adjusting for alcohol use.

Population-attributable risk (PAR) was estimated in the final step of analysis. Population-attributable risk can be used to estimate the potential effect of interventions that alter rates of exposure to risk factors. Population-attributable risk is defined as the proportion of disease incidence in a population of exposed and not-exposed individuals that is due to exposure; therefore, it is the percent by which incidence would decrease if exposure was reduced. Relating these concepts to this paper, PAR is the percent of the incidence of drink/driving at a particular level (i.e., nondrink/drivers, low-drink/drivers high-drink/drivers, and drank while driving) in the population that would be 
eliminated if exposure to risk factors was reduced. This standard definition holds true when studying disease that is caused by exposure to a pathogen. Because it is not known to what extent the observed associations between the risk factors examined in this study and drink/driving are causal, PAR represents the maximum decrease in drink/driving that could be achieved if the risk factors were eliminated.

Population-attributable risk was calculated for variables used in the analyses that could conceptually influence driving behaviors, and that might feasibly be altered by intervention. These variables included perceived risk of drink/driving, social support for drinking and drink/ driving, risk taking, hostility, delinquent behavior, and competitive attitude toward driving. Population-attributable risk was calculated for 2 amounts of change in the drink/driving risk factors just listed: a reduction in exposure such that everyone in the population scored at or below the 75th percentile score for the observed risk factor; and a reduction to the 50th percentile or lower. Population-attributable risk was estimated separately for each of the 6 risk factors just listed and then a combined estimate was calculated for the variables with the highest PAR (i.e., the potential effect of an intervention that reduced levels of more than 1 risk factor simultaneously).

To estimate PAR for a given predictor $X$ of drink/driving group, a model-based estimate of the probability of a given level $d$ of drink/driving for each subject was obtained under the observed predictor and additional set of covariates $Z$, and a similar "future" estimate was obtained by the value of the predictor of interest to the $p$ th percentile level for all cases at which the predictor of interest was above the $p$ th percentile:

$$
x_{i}^{\mathrm{fut}}=\left\{\begin{array}{lll}
x_{i}^{\mathrm{obs}} & \text { if } x_{i}^{\mathrm{obs}} & \leq x_{(p)} \\
x_{(p)} & \text { if } x_{i}^{\mathrm{obs}} & >x_{(p)}
\end{array}\right.
$$

The difference in the average of these probabilities across the sample is an estimate of the attributable risk (AR). An estimate of PAR was then given by 1 minus the ratio of the average predicted probabilities under the "future" model where exposure has been reduced to the average predicted probabilities under the current distribution of exposures:

$$
\begin{aligned}
\mathrm{AR}= & \sum_{i} \hat{P}\left(D D_{i}=d \mid X_{i}=x_{i}, \mathbf{Z}_{i}=\mathbf{z}_{i}\right) / n \\
& -\sum_{i} \hat{P}\left(D D_{i}=d \mid X_{i}=x_{i}^{\mathrm{fut}}, \mathbf{Z}_{i}=\mathbf{z}_{i}\right) / n \\
\mathrm{PAR}= & 1-\frac{\sum_{i} \hat{P}\left(D D_{i}=d \mid X_{i}=x_{i}^{\mathrm{fut}}, \mathbf{Z}_{i}=\mathbf{z}_{i}\right) / n}{\sum_{i} \hat{P}\left(D D_{i}=d \mid X_{i}=x_{i}, \mathbf{Z}_{i}=\mathbf{z}_{i}\right) / n}
\end{aligned}
$$

where under the multinomial logit model,

$$
\begin{aligned}
& \hat{P}\left(D D_{i}=d \mid X_{i}=x_{i}, \mathbf{Z}_{i}=\mathbf{z}_{i}\right)=\frac{\exp \left(\hat{\alpha}^{d}+\hat{\beta}^{d} x_{i}+\hat{\gamma}^{d} \mathbf{z}_{i}\right)}{1+\exp \left(\hat{\alpha}^{d}+\hat{\beta}^{d} x_{i}+\hat{\gamma}^{d} \mathbf{z}_{i}\right)}, \\
& \quad d=1, \ldots, 4
\end{aligned}
$$

Separate PAR estimates were generated for men and women. All estimates of PAR were adjusted for alcohol use. Confidence intervals for PAR were obtained by bootstrapping the results using 2,000 resamplings of the data with replacement.

When PAR estimates are interpreted, it is important to remember that the effect indicated by the PAR value is proportional to the size of the subgroup it is associated with. For example, a given PAR for a group that represents a small proportion of the population represents a smaller overall influence on the outcome for the entire population than the same-sized PAR for a larger population subgroup.

\section{RESULTS}

\section{Descriptive Analyses}

Correlations among the predictor variables were examined, and variance inflation was calculated before conducting any analyses. The bivariate correlations were mostly small (i.e., $r=0.08-0.20$ ) but ranged from $r=0$ to 0.62 . The variance inflation estimates showed no indication of colinearity.

Spearman's correlations between drink/driving group and each of the risk factors unadjusted and adjusted for alcohol use were calculated. The effects of adjusting the correlations for alcohol use generally weakened the association between drink/driving and the risk factors, with alcohol use completely accounting for the associations with drink/driving in only a few risk factors. For women, alcohol use completely accounted for the associations between drink/driving and risk-taking propensity, hostility, cigarette smoking, and safety belt use. For men, alcohol use completely accounted for the associations between drink/driving group and hostility and cigarette smoking. These simple analyses suggest that the association of some risk factors with drink/driving is spurious, arising entirely from the association between drink/driving and alcohol use.

\section{Prediction of Drink/Driving Group}

Two sets of multinomial logistic regression models were tested for men and women. The first set regressed drink/ driving group on each set of variables separately. The second set of analyses regressed drink/driving group onto all the predictors simultaneously. In both sets of analyses, social support for drinking and drink/driving was the strongest predictor of drink/driving group for both men and women, and perceived risk of drink/driving was also a strong predictor for women. 
Significant predictors from the first set of models are shown in Table 1. Generally, adjusting the model for alcohol use decreased the strength of the association between the risk factors and drink/driving. In many cases, the change was small, indicating that alcohol does not account for much of the association between the risk factors and drink/driving when all of the risk factors within the same group are entered. Exceptions for women included tolerance of deviance, social support for drinking and drink/ driving, and delinquent behavior. For men, risk taking, hostility, tolerance of deviance, social support for drinking and drink/driving, and delinquent behavior decreased when the models were adjusted for alcohol use.

Table 2 displays the significant unadjusted and adjusted models when all of the risk factors were entered into the model simultaneously. In these models, only perceived risk of drink/driving, social support for drinking and drink/ driving, delinquent behavior, cigarette smoking, and highrisk driving were significant for women. Cigarette smoking was no longer significant after adjusting for alcohol use, and odds ratios for social support for drinking and drink/ driving, and tolerance of deviance decreased when the model was adjusted for alcohol use. For men, perceived risk of drink/driving, social support for drinking and drink/driving, delinquent behavior, drug/driving, highrisk driving, and safety belt use were significant in the unadjusted model. Safety belt use was no longer significant, and social support for drinking and drink/driving showed a large decrease in strength when the model was adjusted for alcohol use.

In the adjusted models, participants with high social support for drinking and drink/driving were 5.92 (women)

Table 1. Significant Results of Multinomial Logistic Regression Models Tested for Each Content Area Separately: Odds Ratios of Comparisons With the Nondrink/Driver Group

\begin{tabular}{|c|c|c|c|c|c|c|}
\hline \multirow[b]{2}{*}{ Predictor variables } & \multicolumn{3}{|c|}{ Unadjusted } & \multicolumn{3}{|c|}{ Adjusted } \\
\hline & Low-drink/driver & High-drink/driver & $\begin{array}{l}\text { Drink while } \\
\text { driving }\end{array}$ & Low-drink/driver & High-drink/driver & $\begin{array}{l}\text { Drink while } \\
\text { driving }\end{array}$ \\
\hline \multicolumn{7}{|l|}{ Women } \\
\hline \multicolumn{7}{|l|}{ Personality/hostility } \\
\hline Risk-taking propensity & $1.71^{* *}$ & $2.38 * * *$ & $3.69 * * *$ & $1.56^{*}$ & 1.38 & $2.13^{* * *}$ \\
\hline Hostility & 1.14 & 1.01 & 1.63 & 1.12 & $1.70^{* *}$ & $2.26 * * *$ \\
\hline Tolerance of deviance & $2.58 * * *$ & $5.90 * * *$ & $5.88 * * *$ & $1.71^{*}$ & $2.79 * * *$ & $4.19 * * *$ \\
\hline Competitive driving & $1.45^{* *}$ & 1.27 & $1.77^{* *}$ & 1.26 & 1.16 & 1.37 \\
\hline \multicolumn{7}{|l|}{ Individual/social characteristics } \\
\hline Perceived risk of drink/driving & $1.63^{* * *}$ & $2.26^{* * *}$ & $3.10 * * *$ & $1.64 * * *$ & $2.27^{* * *}$ & $3.14 * * *$ \\
\hline Social support for drinking and drink/driving & $2.82^{* * *}$ & $9.50 * * *$ & $15.63^{* * *}$ & $1.85^{* * *}$ & $4.58^{* * *}$ & $6.57^{* * *}$ \\
\hline \multicolumn{7}{|l|}{ Problem behavior } \\
\hline Delinquent behavior & $3.59 * * *$ & $5.20 * * *$ & $10.44^{* * *}$ & $2.76^{* * *}$ & $3.18^{* * *}$ & $5.55^{* * *}$ \\
\hline Lifetime substance use & $1.22^{*}$ & $1.30 * *$ & $1.31^{*}$ & 1.09 & 1.08 & 1.03 \\
\hline Past-year substance use & $1.33^{*}$ & $1.88 * * *$ & $2.20 * * *$ & 1.15 & $1.47^{*}$ & $1.74^{* *}$ \\
\hline Cigarette smoking & $1.02^{*}$ & $1.03^{* *}$ & 1.00 & 1.01 & 1.02 & 0.98 \\
\hline \multicolumn{7}{|l|}{ Driving behavior } \\
\hline Drug/driving & $1.10^{*}$ & $1.23^{* * *}$ & $1.31 * * *$ & 1.05 & $1.12^{* *}$ & $1.19 * * *$ \\
\hline High-risk driving & $1.31^{* * *}$ & $1.65^{* *}$ & $1.65 * * *$ & $1.25^{* * *}$ & $1.53^{* * *}$ & $1.48 * * *$ \\
\hline Risk-taking driving & 0.79 & 1.72 & 1.31 & 0.90 & 2.22 & 1.01 \\
\hline Aggressive driving & 1.26 & 1.33 & 1.33 & 1.15 & 1.12 & 1.05 \\
\hline Safety belt use & 0.99 & 1.05 & $1.49^{*}$ & 1.01 & 1.10 & $1.61^{* * *}$ \\
\hline \multicolumn{7}{|l|}{ Men } \\
\hline \multicolumn{7}{|l|}{ Personality/hostility } \\
\hline Risk-taking & $1.56^{*}$ & 1.38 & $2.13^{* * *}$ & 1.41 & 1.06 & 1.47 \\
\hline Hostility & 1.12 & $1.70^{* *}$ & $2.26 * * *$ & 0.92 & 1.02 & 1.11 \\
\hline Tolerance of deviance & $1.71^{*}$ & $2.80 * * *$ & $4.18^{* * *}$ & 1.54 & $2.26^{* *}$ & $2.72 * * *$ \\
\hline Competitive driving & 1.26 & 1.16 & $1.37^{*}$ & 1.24 & 1.07 & 1.18 \\
\hline \multicolumn{7}{|l|}{ Individual/social characteristics } \\
\hline Perceived risk of drink/driving & $1.46^{* * *}$ & $1.81^{* * *}$ & $1.73 * * *$ & $1.49 * * *$ & $1.85 * * *$ & $1.84^{* * *}$ \\
\hline $\begin{array}{l}\text { Social support for drinking and } \\
\text { drink/driving }\end{array}$ & $2.02^{* * *}$ & $7.91^{* * *}$ & $20.72^{* * *}$ & $1.67^{* * *}$ & $4.62^{* * *}$ & $9.22^{* * *}$ \\
\hline \multicolumn{7}{|l|}{ Problem behavior } \\
\hline Delinquent behavior & $2.34^{* * *}$ & $4.27^{* * *}$ & $9.82 * * *$ & $1.85^{*}$ & $2.86^{* * *}$ & $5.13^{* * *}$ \\
\hline Lifetime substance use & 1.11 & 1.08 & 1.04 & 1.02 & 0.91 & 0.84 \\
\hline Past-year substance use & $1.19^{* * *}$ & $1.84^{* * *}$ & $2.42^{* * *}$ & 1.11 & $1.54^{* *}$ & $1.90 * * *$ \\
\hline Cigarette smoking & 1.00 & 1.02 & 1.01 & 0.99 & 1.00 & 1.00 \\
\hline \multicolumn{7}{|l|}{ Driving behavior } \\
\hline Drug/driving & 1.04 & $1.16^{* * *}$ & $1.26 * * *$ & 1.02 & $1.10^{* *}$ & $1.16 * * *$ \\
\hline High-risk driving & $1.26^{* * *}$ & $1.60 * * *$ & $1.81^{* * *}$ & $1.23^{* * *}$ & $1.55^{* * *}$ & $1.72^{* * *}$ \\
\hline Risk-taking driving & 0.79 & $1.82^{*}$ & 1.43 & 0.83 & $2.08^{*}$ & 1.75 \\
\hline Aggressive driving & 1.20 & 1.07 & 1.16 & 1.13 & 0.91 & 0.90 \\
\hline Safety belt use & 0.89 & $1.22 *$ & $1.72^{* * *}$ & 0.88 & 1.18 & $1.58^{* * *}$ \\
\hline
\end{tabular}

${ }^{*} p<0.05 ; *{ }^{*} \leq 0.01 ; * * p \leq 0.001$. 
Table 2. Significant Results of Multinomial Logistic Regression Model With All Predictors Entered Together: Odds Ratios of Significant Comparisons With the NDD Group

\begin{tabular}{|c|c|c|c|c|c|c|}
\hline \multirow[b]{2}{*}{ Predictor variables } & \multicolumn{3}{|c|}{ Unadjusted } & \multicolumn{3}{|c|}{ Adjusted } \\
\hline & Low-drink/driver & High-drink/driver & $\begin{array}{l}\text { Drink while } \\
\text { driving }\end{array}$ & Low-drink/driver & High-drink/driver & $\begin{array}{l}\text { Drink while } \\
\text { driving }\end{array}$ \\
\hline \multicolumn{7}{|l|}{$\begin{array}{l}\text { Women } \\
\text { Individual/social characteristics }\end{array}$} \\
\hline Perceived risk of drink/driving & $1.73^{* * *}$ & $2.32^{* * *}$ & $2.86^{* * *}$ & $1.74^{* * *}$ & $2.38 * * *$ & $2.91 * * *$ \\
\hline Social support for drinking and drink/driving & $2.70^{* * *}$ & $9.07^{* * *}$ & $11.57^{* * *}$ & $1.97^{* * *}$ & $5.52^{* * *}$ & $5.92^{* * *}$ \\
\hline \multicolumn{7}{|l|}{ Problem behavior } \\
\hline Delinquent behavior & $2.75^{* * *}$ & 1.83 & $5.02 * *$ & $2.50 * *$ & 1.53 & $3.89^{*}$ \\
\hline Cigarette smoking & 1.01 & $1.03^{*}$ & 0.99 & - $^{\mathrm{a}}$ & - & - \\
\hline \multicolumn{7}{|l|}{ Driving behavior } \\
\hline High-risk driving & $1.21 * * *$ & $1.52^{* * *}$ & $1.37^{* *}$ & $1.18^{* *}$ & $1.49 * * *$ & $1.31^{*}$ \\
\hline Men & & & & & & \\
\hline \multicolumn{7}{|l|}{ Individual/social characteristics } \\
\hline Perceive risk of drink/driving & $1.45^{* *}$ & $1.82^{* * *}$ & $1.58^{* *}$ & $1.49^{* * *}$ & $1.88 * * *$ & $1.67^{* *}$ \\
\hline Social support for drinking and drink/driving & $2.08^{* * *}$ & $7.62^{* * *}$ & $18.19^{* * *}$ & $1.84^{* * *}$ & $5.16 * * *$ & $9.93^{* * *}$ \\
\hline Problem behavior & & & & & & \\
\hline Delinquent behavior & 1.44 & 1.85 & $4.17^{* * *}$ & 1.32 & 1.62 & $3.07^{* *}$ \\
\hline \multicolumn{7}{|l|}{ Driving behavior } \\
\hline Drug/driving & 1.01 & 1.05 & $1.14^{*}$ & 1.00 & 1.03 & $1.12^{*}$ \\
\hline High-risk driving & $1.21 * *$ & $1.51^{* * *}$ & $1.63^{* * *}$ & $1.20 * *$ & $1.51 * * *$ & $1.61^{* * *}$ \\
\hline Safety belt use & $0.78^{*}$ & 0.92 & 1.23 & - $^{\mathrm{a}}$ & - & - \\
\hline
\end{tabular}

${ }^{*} p<0.05 ;{ }^{* *} \leq 0.01 ;{ }^{* * *} p \leq 0.001 .{ }^{a}$ This variable did not enter the model adjusted for alcohol use.

and 9.93 (men) times more likely to be in the drank while driving group than the nondrink/drivers group, and 5.52 (women) and 5.16 (men) times more likely to be in the highdrink/drivers than the nondrink/drivers group. Women and men with low social support for drinking and drink/driving were 1.97 and 1.84 times more likely to be in the low-drink/ drivers than the nondrink/drivers group. Women with high perceived risk of drink/driving were 2.91, 2.38, and 1.74 times more likely to be in the drank while driving, highdrink/drivers, and low-drink/drivers groups than the nondrink/drivers group, respectively, and for men, the odds were 1.67, 1.88, and 1.49, respectively. Delinquent behavior was also a strong predictor for women.

\section{Population-Attributable Risk}

The PAR calculations generally indicated that perceived risk of drink/driving contributed more to drink/driving among women than men, and that interventions that reduce perceived risk of drink/driving may be more effective for women. Population-attributable risk for social support for drinking and drink/driving was large for both men and women, suggesting that interventions that reduce individual social support for drinking and drink/ driving may potentially have a large effect for both sexes, with reductions in drink/driving of up to $33 \%$ for men and women in the drank while driving group. Clearly, when PAR is calculated for both perceived risk of drink/driving and social support for drinking and drink/driving simultaneously, the PAR values are the largest, with reductions in drink/driving of up to $56 \%$ for women and $33 \%$ for men if an intervention reduced both perceived risk of drink/ driving and social support for drinking and drink/driving to the level of the median for this sample.

\section{DISCUSSION}

Alcohol consumption and drink/driving are strongly associated. In addition, these 2 variables share many of the same predictors and risk factors. Past research has largely ignored the potential confounding nature of these associations, and little effort has been made to determine the extent to which the association between known risk factors and drink/driving is due to alcohol use and its association with drink/driving, as opposed to unique variance associated with drink/driving, per se. Conceptually, one would anticipate 1 of 2 types of associations: one that is completely confounded by the association between alcohol use and drink/driving, and one in which this association only partially confounds the results. In the former situation, when models testing the association are adjusted for alcohol use, there would no longer be a significant association between these risk factors and drink/ driving, and second, an association in which only partial confounding occurs. In this type of association, significant effects would be diminished but remain significant when models were adjusted for alcohol use.

The results of this study identify the partial and complete confounding effects that broaden our understanding of drink/driving. In particular, these results suggest that the associations between drink/driving and personality/ hostility factors, substance use, and drug/driving are due to these latter variables' associations with level of alcohol use, and that the associations between drink/driving and essentially all the variables studied are at least partially 
confounded by alcohol use. These results support the hypothesis that some factors that have been identified as indicative of risk for drink/driving are actually unique to alcohol use, and do not necessarily contribute to drink/ driving. These results begin to shed clearer light on the characteristics of individuals that are related specifically to their drink/driving behavior, and provides some initial ideas regarding how drink/driving behavior might change if certain individual characteristics are modified through intervention and/or individual treatment.

The results of this study contribute some insight and understanding of the characteristics that distinguish drink/ drivers from nondrink/drivers, and will be useful in identifying individuals at risk for drink/driving. This increased understanding has significant value as basic knowledge, opening the door for investigations of the processes and mechanics involved in the occurrence of drink/driving. Additionally, this information has practical importance for the development of programs, interventions, and policies to prevent drinking while driving. Specifically, the results of the PAR analyses indicate that, given the measures included in this study, preventive interventions would be effective if they targeted either perceived risk of drink/ driving, or social support for drink/driving, and that maximum effectiveness would result from addressing both. Regression results showed that the associations of these 2 measures with drink/driving are largely independent of each other, which contributes to a combined PAR value, suggesting that interventions that reduce both these variables to the median level would decrease drink/driving by as much as $56 \%$ for women and $33 \%$ for men. Although the size of the effects resulting from interventions that reduce these 2 characteristics depend on the extent to which the association of these 2 variables with drink/ driving is causal, they clearly indicate 2 areas where interventions might have a large effect.

Future research on this topic should address the limitations of this study by examining the issues addressed here using a broader set of variables than were available for this study, in more diverse samples, and in other populations, so that additional correlates of drink/driving can be identified, and thus knowledge of which variables uniquely influence drink/driving can be expanded and their interactions elucidated. Specifically, variables that can be modified by interventions should be identified, so that the effectiveness of efforts to reduce drink/driving can be increased. Longitudinal research examining the development of drink/driving behavior as it relates to the unique predictors of this behavior would also provide critical information in the prevention of drink/driving, and perhaps identify critical points at which interventions would be especially effective. Finally, research needs to connect person-level and ecological-level variables that contribute to drink/driving. An examination of these factors may provide an insight into the connections between individual characteristics and the drinking loca- tions and setting that they choose. Given the association between drinking location and drink/driving (Gruenewald et al., 1996), an understanding of the variables that bridge person and context could contribute significantly to efforts to reduce drink/driving.

\section{REFERENCES}

American Association for Public Opinion Research (2000) Standard definitions: final dispositions of case codes and outcome rates for surveys. Available at: http://www.aapor.org/default.asp?page= survey_methods/ standards_and_best_practices/standard_definitions. Accessed September 21, 2006

Babor TF, de la Fuente JR, Saunders J, Grant M (1992) AUDIT: The Alcohol Use Disorders Identification Test. Guidelines for Use in Primary Health Care. World Health Organization, Geneva.

Babor TF, Del Boca FK (1992) Just the facts: enhancing measurements of alcohol consumption using self-report methods, in Measuring Alcohol Consumption (Litten R, Allen J eds), pp. 3-20. Humana Press, Totawa, NJ.

Babor TF, Steinberg K, Anton R, Del Boca FK (2000) Talk is cheap: measuring drinking outcomes in clinical trials. J Stud Alcohol 61: 55-63.

Baum S (2000) Drink driving as a social problem: comparing the attitudes and knowledge of drink driving offenders and the general community. Accid Anal Prev 32:689-694.

Bingham CR, Shope JT (2004) Adolescent problem behavior and problem driving in young adulthood. J Adol Res 19:205-223.

Burns PC, Wilde GJS (1995) Risk taking in male taxi drivers: relationships among personality, observational data and driver records. Person Indiv Diff 18:267-278.

Cattell RB (1982) The psychometry of objective motivation measurement: a response to the critique of Cooper and Kline. Br J Educ Psychol 52:234-241.

Chaikelson JS, Arbuckle T, Lapidus S, Gold D (1994) Measurement of lifetime alcohol consumption. J Stud Alcohol 55:133-140.

Cohen J (1992) A power primer. Psychol Bull 112:155-159.

Darke S, Heather N, Hall W, Ward J, Wodak A (1991) Estimating drug consumption in opioid users: reliability and validity of a 'recent use' episodes method. Br J Addict 86:1311-1316.

Donovan DM, Queisser HR, Salzberg PM, Umlauf R (1985) Intoxicated and bad drivers: subgroups within the same population of high-risk men drivers. J Stud Alcohol 46:375-382.

Donovan JE (1993) Young adult drink-driving: behavioral and psychosocial correlates. J Stud Alcohol 54:600-613.

Elliott MR, Waller PF, Raghunathan TE, Shope JT, Little RJA (2000) Persistence of violation and crash behavior over time. J Saf Res 31:229-242.

Escobedo LG, Chorba TI, Waxweiler R (1995) Patterns of alcohol-use and the risk of drinking and driving among us high-school-students. Am J Public Health 85:976-978.

Everett SA, Lowry R, Cohen LR, Dellinger AM (1999) Unsafe motor vehicle practices among substance-using college students. Accid Anal Prev 31:667-673.

Grasmick HG, Bursik RJ, Arneklev BJ (1993) Reduction in drunk driving as a response to increased threats of shame, embarrassment, and legal sanctions. Criminology 31:41-67.

Greenberg MD, Morral AR, Jain AK (2005) Drink-driving and DUI recidivists' attitudes and beliefs: a longitudinal analysis. J Stud Alcohol 66:640-647.

Greenfield TK, Rogers JD (1999) Alcoholic beverage choice, risk perception, and self-reported drunk driving: effects of measurement on risk analysis. Addiction 94:1735-1743.

Grube JW, Voas RB (1996) Predicting underage drinking and driving behaviors. Addiction 91:1843-1858. 
Gruenewald PJ, Mitchell PR, Treno AJ (1996) Drinking and driving: drinking patterns and drinking problems. Addiction 91:1637-1649.

Howland J, Hingson R (1987) Alcohol as a risk factor for injuries or death due to fires and burns - a review of the literature. Public Health Rep 102:475-483.

Iversen H, Rundmo T (2002) Personality, risky driving and accident involvement among Norwegian drivers. Persona Indiv Diff 33:12511263.

Jonah BA (1997) Sensation seeking and risky driving: a review and synthesis of the literature. Accid Anal Prev 29:651-665.

Lee JA, Jones-Webb RJ, Short BJ, Wagenaar AC (1997) Drinking location and risk of alcohol-impaired driving among high school seniors. Addict Behav 22:387-393.

McMillen DI, Pang MG, Wells-Parker E, Anderson BJ (1991) Behavior and personality-traits among DUI arrestees, nonarrested impaired drivers, and nonimpaired drivers. Int J Addict 26:227-235.

McMillen DI, Pang MG, Wells-Parker E, Anderson BJ (1992) Alcohol, personality-traits, and high-risk driving - A comparison of young, drinking driver groups. Addict Behav 17:525-532.

National Highway Traffic Safety Administration (2005). Traffic Safety Facts 2004 Data: Alcohol. DOT HS 809 905. Available at: http:// www-nrd.nhtsa.dot.gov/pdf/nrd-30/NCSA/TSF2004/809905.pdf

O'Malley PM, Bachman JG, Johnston LD (1983) Reliability and consistency in self-reports of drug use. Int J Addict 18:805-824.

Padilla A, Morrisey L (1993) Place of last drink by repeat DUI offenders: a retrospective study of gender and ethnic group differences. Hispanic J Behav Sci 15:357-372.

Patil SM, Shope JT, Raghunathan TE, Bingham CR (2006) The role of personality characteristics in young adult high-risk driving. Traffic Injury Prev, 7:1-8.

Sabel JC, Bensley LS, vanEenwyk J (2004) Associations between adolescent drinking and driving involvement and self-reported risk and protective factors in students in public schools in Washington state. J Stud Alcohol 65:213-216.

Shepherd J, Farrington D, Potts J (2004) Impact of antisocial lifestyle on health. J Public Health 26:347-352.

Shope JT, Bingham CR (2002) Drinking-driving as a component of problem driving and problem behavior in young adults. J Stud Alcohol 63:24-33.
Shope JT, Copeland LA, Marcoux BC, Kamp ME (1996) Effectiveness of a school-based substance abuse prevention program. J Drug Educ 26:323-337.

Shope JT, Dielman TE, Butchart AT, Campanelli PC (1992) An elementary school-based alcohol misuse prevention program: follow-up evaluation. J Stud Alcohol 53:106-120.

Shope JT, Elliott MR, Raghunathan TE, Waller PF (2001) Long-term follow-up of a high school alcohol misuse prevention program's effect on students' subsequent driving. Alcohol Clin Exp Res 25: 403-410.

Treno AJ, Gruenewald PJ, Ponicki WR (1996) Use of ICD-9-CM codes in the estimation of alcohol-involved injury: search for a surrogate II. Alcohol Clin Exp Res 20:320-326.

Turrisi R, Jaccard J, McDonnell D (1997) An examination of the relationships between personality, attitudes, and cognitions relevant to alcohol-impaired driving tendencies. J Appl Soc Psychol 27: 1367-1394.

Ulleberg P (2001) Personality subtypes of young drivers. Relationships to risk-taking preferences, accident involvement, and response to a traffic safety campaign. Transp Res F Traffic Psychol Behav 4: 279-297.

Ulleberg P, Rundmo T (2003) Personality, attitudes and risk perception as predictors of risky driving behaviour among young drivers. Saf Sci 41:427-443.

Vingilis E, Stoduto G, Macartney-Filgate MS, Liban CB, McLellan BA (1994) Psychosocial characteristics of alcohol-involved and nonalcohol-involved seriously injured drivers. Accid Anal Prev 26: 195-206.

Williams AF (2006) Alcohol-impaired driving and its consequences in the United States: the past 25 years. J Saf Res 37:123-138.

Williams CL, Toomey TL, McGovern P, Wagenaar AC, Perry CL (1995) Development, reliability, and validity of self report alcohol-use measures with young adolescents. J Child Adolescent Subst Abuse $4: 17-40$.

Yu J, Evans PC, Perfetti L (2004) Road aggression among drinking drivers: alcohol and non-alcohol effects on aggressive driving and road rage. J Crim Justice 32:421-430. 